\title{
Mecanismo, función, expresión y narración: la cuádruple interpretación de las emociones, una versión desde el pragmatismo*
}

Martha Jeaneth Patiño Barragán**

Artículo de investigación sobre los distintos modos como se ha interpretado el fenómeno de las emociones, partiendo de Aristóteles para evidenciar cómo en el pragmatismo norteamericano de James y Dewey ocurre una bifurcación de las explicaciones que deriva, la de James, en la explicación de la neurociencia; y la de Dewey, en la interpretación de las ciencias sociales.

Recibido: 12 de marzo de 2020

Evaluado: 31 de julio de 2020

Aceptado: 16 de diciembre de 2020

Publicado: 1 de julio de 2021

Citar como:

Patiño, M. (2021). Mecanismo, función, expresión y narración: la cuádruple interpretación de las emociones, una versión desde el pragmatismo. Hallazgos, 18(36), 135-178. https://doi.org/10.15332/2422409X.5026

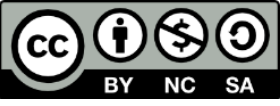

\footnotetext{
* Artículo presentado como resultado de investigación para el proyecto institucional INV-HUM 2606 titulado: Neurociencia, educación y emociones, fase 1, financiado por la Vicerrectoría de Investigaciones de la Universidad Militar Nueva Granada, Colombia. ** Doctora en Filosofía. Docente de planta e investigadora, Facultad de Ingeniería, Universidad Militar Nueva Granada, Colombia.

Correo electrónico: martha.patino@unimilitar.edu.co

ORCID: https://orcid.org/0000-0002-1467-8889
}

Hallazgos

ISSN: 1794-3841 | e-ISSN: 2422-409X | DOI: https://doi.org/10.15332/2422409X

Vol. 18 N.o 36| julio-diciembre del 2021 


\section{Resumen}

Este artículo tiene por objeto mostrar una lectura sobre el problema de las emociones, de la interacción entre los abordajes de la filosofía y sus derivaciones y diálogos con otras discipinas. Para tal fin, se toma como punto central la influencia que William James y John Dewey, desde el pragmatimo clásico, han ejercido en los desarrollos contemporáneos como el cognitivismo, así como en aquellos de las ciencias sociales y en la narrativa individual de la experiencia. No obstante, para conectar estos abordajes metodológicamente se parte de la versión aristotélica, la cual da origen a los diferentes enfoques actuales.

Palabras clave: Cognitivismo y emociones; Emociones; Emociones en el pragmatismo clásico; Emociones en filosofía; Emociones en las ciencias sociales; Emociones y neurociencia.

\section{Mechanism, function, expression and narration: the fourfold interpretation of emotions, a version from pragmatism}

\section{Abstract}

This article aims to show a reading on the problem of emotions, of the interaction between the approaches of philosophy and its derivations and dialogues with other disciplines. To this end, the main theme of the article revolves around the influence that William James and John Dewey, from the classical pragmatism, have exerted on contemporary developments such as cognitivism, as well as on those of the social sciences and on individual narrative of experience. However, in order to connect these approaches methodologically, we start from the Aristotelian version, which gives rise to the different current approaches. Keywords: Cognitivism and emotions; Emotions; Emotions in classical pragmatism; Emotions in philosophy; Emotions in social sciences;

Emotions and neuroscience. 


\section{Mecanismo, função, expressão e narração: a interpretação quádrupla das emoções, uma versão a partir do pragmatismo}

\section{Resumo}

Este artigo tem como objetivo apresentar uma leitura sobre a problemática das emoções, da interação entre as abordagens da filosofia e suas derivações e diálogos com outras disciplinas. Com essa finalidade, toma-se como ponto central a influência que William James e John Dewey, do pragmatismo clássico, têm exercido sobre desenvolvimentos contemporâneos como o cognitivismo, bem como no andamento das ciências sociais e da narrativa individual da experiência. No entanto, para conectar essas abordagens metodologicamente, partimos da versão aristotélica, que dá origem às diferentes abordagens atuais.

Palavras-chave: Cognitivismo e emoções; Emoções; Emoções no pragmatismo clássico; Emoções em filosofia; Emoções nas ciências sociais; Emoções e neurociência.

\section{Introducción}

Cada uno de los acercamientos al problema de las emociones constituye un gran aporte a la comprensión de su complejidad. En esta línea, en el presente artículo se tiene la intención de mostrar la importancia de la herencia filosófica, especialmente del pragmatismo clásico, en su aporte a las neurociencias, las ciencias sociales y las singularidades narrativas que describen según la experiencia la vivencia de las emociones, de manera que puedan ser comprendidas en su dimensión compleja, e incluyan su aspecto visible, exterior, mecánico, y su aspecto interior, espiritual, cultural, así como la narrativa individual.

Se trata de poner de presente la influencia que en otras disciplinas - psicología, neurociencias, ciencias sociales - han tenido las teorías de William James y John Dewey, quienes, a pesar de ser pragmatistas, 
difieren bastante en el tratamiento del problema. Si bien ambos son referente, lo son de disciplinas distintas: James, de la psicología y las neurociencias, y Dewey, de las ciencias sociales. Claramente, James ocupa un lugar privilegiado en el abordaje del problema. De su teoría se derivan varios estudios importantes durante gran parte del siglo XX. Tal fenómeno obedece a la particular inclinación de esta época a enfocar las emociones desde el punto de vista de una "racionalidad objetiva". Se intenta aquí presentar algunos de los motivos que pudieron haber llevado a las elecciones hechas de acuerdo con la herencia de James, para que el relato que las entiende como mecanismo físico causal haya logrado este esplendor.

John Dewey, por su parte, al ocuparse de explicar la emoción como expresión cargada de sentido dentro de un marco de significación, tanto cultural como individualmente, influye y dialoga sobre todo con las ciencias sociales en lo que tiene que ver con el aspecto expresivo de la emoción. El aporte del presente artículo a este recorrido muestra otro aspecto derivado de Dewey pero perfeccionado por Rorty (1991), y que poco se tiene en cuenta en filosofía: el de darle un lugar también a la narración individual de la emoción, expresada mediante el relato particular, a fin de pasar de mirar desde afuera a escuchar lo que ocurre dentro de los individuos y de su experiencia subjetiva vivida.

\section{Metodología}

La delimitación del tema se define con base en la pregunta por la transformación del concepto emoción y la necesidad de tener en cuenta otros relatos para componer el mosaico de su sofisticado acaecer. Para lograr dicho objetivo se realiza una revisión sistemática de textos, específicamente fuentes primarias, con un criterio histórico que muestre la transformación de las representaciones e interpretaciones, a fin de resaltar 
las distintas teorías relacionadas con la concepción de su naturaleza. De este modo, se busca mostrar las interconexiones con otras disciplinas, y proponer la otra visión inspirada en Dewey que no se ha considerado como parte de la discusión.

Se recogen los estudios disponibles, en español e inglés, en formato de artículos críticos y libros de comentaristas, según las siguientes categorías: filosofía de la emoción, emociones y neurociencia, emociones en las ciencias sociales, emociones en el pragmatismo clásico y emociones y narrativa. Desde estas se pueden abordar los comentaristas de la historia del problema mediante búsquedas en bases de datos. Luego se escogen los autores que han trabajado el problema, y, por último, este se delimita a las interpretaciones para responder a la pregunta por su sentido y la ausencia de un elemento fundamental, a saber: la experiencia individual no patologizada.

\section{A modo de contexto}

En filosofía de las emociones hay tres perspectivas claramente demarcadas. La primera sostiene que las emociones son impulsos básicos que emergen como resultado de procesos corporales. En esta concepción anidan las teorías biologicista y fisiologicista, y aquella tradición que asegura que las emociones son fenómenos sofisticados y significativos que se originan en los pensamientos, es decir, en la mente, también llamados cogniciones. Se destacan, además, las concepciones socioconstructivistas de Averil (1980) y Mesquita et ál. (2016), que hablan de las emociones como construcciones sociales. Estos distintos planteamientos se arraigan principalmente en filosofía. Aristóteles, quien entendió las emociones desde un punto de vista holista como expresiones humanas complejas, tanto físicas como mentales con efecto en las acciones, las cuales son sociales, no profundizó demasiado en ellas, lo que sí ha hecho la filosofía 
contemporánea. Vale decir, no obstante, que su inquietud y descripción acerca de las emociones ha sido un aporte significativo a la discusión contemporánea, sobre todo como referente en cuanto a definición y comprensión no reduccionistas. Por eso en la siguiente sección se expone su perspectiva, a fin de mostrar cómo los distintos posicionamientos subsiguientes retoman sus definiciones y preguntas.

Por su parte, la versión deweyana se acerca, de algún modo, a las teorías de las ciencias sociales, o se inspira en ellas, de acuerdo con su expresividad, así como las apuestas por su experiencia vivida que se manifiestan en narraciones. En este sentido, las emociones son un fenómeno complejo - lo que no significa que no puedan comprenderse- que debe abordarse teniendo en cuenta la mayor cantidad de aristas, sin reduccionismos, desde distintos flancos y disciplinas, de modo que se pueda tener una visión de conjunto. Se elige este camino para mostrar cómo desde sus orígenes las emociones fueron representadas con base en una visión compleja, y cómo por el camino se fueron reduciendo a un mero mecanismo físico-causal, abordado desde la objetividad, referida a las mediciones. De aquí se ha derivado a los estudios de las neurociencias. Esta mirada las analiza según su estructura neurofuncional (sinapsis, neurotransmisores, pautas de ondas electroencefalográficas, etc.), y constituye una "racionalidad objetiva en sus presupuestos esenciales [...] que reduce lo real a un cúmulo de objetos" (Patiño, 2018, p. 90).

Sin embargo, el cognitivismo ha recuperado parte de su complejidad y se ha consolidado como el escenario donde se da cita el debate contemporáneo. Aun así, el mapa no se completa hasta que no se incluyen comprensiones que enriquecen su estudio de acuerdo con la experiencia y la interacción de las subjetividades en contextos históricos y locales socialmente dinámicos, desde las ciencias sociales. Por ello es menester hacer todo el recorrido para así identificar el diálogo interdisciplinar 
necesario que permita tener el mapa completo de los distintos relatos sobre este fenómeno humano. En este sentido, es preciso incluir el relato de las distintas experiencias, ya que estos relatos son versiones fenomenológicamente distintas, y, por ende, “enriquecen la comprensión [...] y muestran las diferentes aristas y sentidos [...] para reconocer las diferentes texturas de lo real” (Patiño, 2018, p. 92).

En el texto se encontrarán los distintos modos de representación del fenómeno propiamente, habida cuenta que a lo largo de la tradición occidental estas se han explicado desde alguno de los aspectos que las constituyen, a saber: el aspecto material - del cual surgen las teorías del sentimiento o sensación o fisiológica -, su rasgo observable o manifestación externa - desde donde se explican como fenómenos universales, lo que origina a variadas teorías conductuales-, desde la versión que las explica como fenómenos mentales - de donde surge la muy extendida visión cognitivo-evaluativa, según la cual las emociones se basan en actos de pensamiento, o son tipos de cognición, o tipos de juicios de valor, que caen dentro de la categoría de creencias, juicios y percepciones, que permite el surgimiento de las teorías del mismo nombre, y que comprende múltiples interpretaciones-. Cada una de estas formas de comprensión da lugar a una u otra teoría que intenta comprender el fenómeno desde un solo aspecto.

Cabe decir que en filosofía las emociones no se tuvieron en cuenta salvo para considerarlas en su aspecto negativo. Esto tuvo que ver con el hecho de que se entendieron como el rasgo humano que debía mantenerse bajo control "con la sabiduría de la razón firmemente en control y los peligrosos impulsos de la emoción suprimidos, canalizados o (idealmente) en armonía con la razón" (Solomon, 2008, p. 3). Las diferentes formas en que la filosofía pregunta por la emoción: ¿̇ué es la emoción?, ¿cuál es su naturaleza?, ¿cuál es su estructura?, e incluso ¿̇existe la emoción?, han 
encontrado de nuevo en el siglo XX un terreno fructífero. Si bien la emoción es parte fundamental de la intimidad de quien la experimenta, es un fenómeno esencialmente subjetivo. Los estudios sobre la emoción en todos los campos apuestan por el criterio de lo observable.

James funda toda una herencia acerca del modo de preguntar y abordar las emociones, que toma fuerza por cuenta de lo que se denomina en este texto como cerebrocentrismo. Aquí se pretende poner en escena otro de los pragmatistas clásicos más importantes, John Dewey, para mostrar su perspectiva de manera que se pueda evidenciar claramente el rumbo que toman sus ideas y su influencia en las ciencias sociales. Es importante aquí hacer el recorrido por la línea de origen de ambos pensamientos, y necesariamente reconocer las tres formas de Aristóteles. Se inicia, entonces, con la versión aristotélica de la unidad cuerpo-mente, para culminar con la teoría deweyana de la emoción, la cual retorna a esta unidad.

\section{Aristóteles: la triple representación de la emoción o el prejuicio de la emoción como esclava de la razón}

Aristóteles es el filósofo que aborda el problema desde tres escenarios en los cuales se manifiesta la emoción. La considera como posibilidad para formar ciudadanos políticos. Para ello se vale de la descripción del fenómeno, de cómo transformarlo según la razón y cómo esta causalidad puede tener efecto en la conducta de los demás. En resumidas cuentas, su intención es principalmente la manipulación de la emoción del otro para lograr fines políticos.

Aristóteles expone las primeras descripciones sobre la emoción, y aunque en filosofía no se atribuye a su estudio la suficiente importancia, su consideración es importante porque las entiende como una sola unidad (Calhoun y Solomon, 1996, p. 11) que se desenvuelve en diferentes 
ámbitos. Según Solomon (Calhoun y Solomon, 1996), en el estudio de este tema, Aristóteles, al articular mente, cuerpo y conducta en una sola experiencia, evita la mayoría de las dificultades surgidas como resultado de muchas teorías, especialmente de corte dualista, porque considera su naturaleza, origen y expresión, de modo que no deja por fuera ninguna de sus facetas. Por el contrario, las concepciones contemporáneas se abordan de manera unívoca, puesto que se presentan demasiadas dificultades de orden ontológico, fenomenológico y conductual, por cuanto defienden uno u otro ámbito como el origen de la emoción, derivado de una cierta definición parcial.

\section{La unidad cuerpo-mente}

En Acerca del alma (1992), Aristóteles señala que el alma racional y el alma animal se conjugan en la aparición de las emociones, tanto en su aspecto cognitivo como en el físico. Esta aproximación permite entenderlas no como impulsos brutos, sino como resultado de procesos en los cuales interviene la razón por medio de creencias, valoraciones, juicios, etc., lo que genera cambios corporales. $\mathrm{Al}$ reconocer el aspecto cognitivo, su relación con la sensación y la utilidad de las emociones considera la posibilidad de controlarlas racionalmente:

Es posible reconocer el modo como interpreta el mundo una persona que se encuentra bajo los efectos de la ira y sobre todo, qué es lo que está pensando en esos momentos acerca de la o las personas hacia las cuales se dirige esta emoción y seguidamente se examina el motivo del enojo, entonces se habrá podido aislar la causa. Una vez reconocida dicha causa, es posible motivar el enojo en otros. (Aristóteles, 1992, p. 10)

Esta inseparabilidad entre creencias, movimientos corporales y cambios fisiológicos cuando se produce una emoción, se aparta de la taxativa distinción "entre los elementos racionales (o cognoscitivos) de la emoción 
y los irracionales (o físicos), reconociendo que las emociones pueden ser ambas cosas en diferentes grados de complejidad". Por esta razón, Calhoun y Solomon (1996) afirman que Aristóteles "evita tratar las emociones como respuestas irracionales e incontroladas a las situaciones" (p. 52), para verlas más bien como la conjugación simultánea de estos tres ámbitos.

Así mismo, el aspecto social-conductual de las emociones es descrito y explicado por Aristóteles, tanto en la Ética (1993) como en la Política (1998). En estas obras da por hecho que los seres humanos son racionales y de naturaleza social, por lo que una buena vida humana implica el desarrollo de habilidades racionales y la participación en diversas formas de vida social (Aristóteles, 1993). Sin embargo, aunque la razón ocupe un preponderante lugar, según Knuutila (2004), en Aristóteles las emociones no se presentan como una agencia separada, puesto que la capacidad para actuar está definida por el modo de sentir las emociones correctas en las circunstancias correctas. Señala Dabrowski (2016) que el sistema de emociones en Aristóteles configura el mapa de valores, interpretaciones y representaciones del mundo, en la medida en que sea posible analizar su contenido cognitivo, que está acompañado por experiencias fisiológicas concretas, las cuales derivan en la acción. Por esta razón se afirma que Aristóteles presenta una de las teorías más completas de las emociones al considerar los elementos cognitivo (evaluación), conductual y fisiológico. Por otro lado, Aristóteles (1990) señala en la Retórica la importancia de los efectos del discurso en las emociones de las personas, "pues las cosas no son, desde luego, iguales para el que siente amistad, que para el que experimenta odio ni para el que está irado que para el que tiene calma" (p. 309). Por lo tanto, "el orador debe inclinar con su discurso a los oyentes" (p. 321). En este sentido, tiene claro Aristóteles que la educación de la juventud debe concentrarse en formar su carácter voluble e 
inconstante (Gadotti, 1998, p. 27), puesto que las emociones afectan el juicio y son la causa de la volubilidad juvenil, de manera que proporciona un consejo útil para los oradores que consiste en cambiar las emociones para cambiar las convicciones de los demás. Al respecto, resalta la importancia de estar en una cierta actitud para que el orador persuada mediante las emociones.

De este modo, su pregunta relevante sería la de cómo entrenar e instruir a los jóvenes a unirse a los patrones emocionales de la cultura, de tal manera que los hábitos de sentimientos y emociones puedan contribuir a una buena vida (Knuutila, 2004, p. 25). Se puede colegir de lo anterior que su estudio de las emociones está orientado a un ejercicio pedagógico del reconocimiento del componente cognitivo que las precede, y a partir de allí, de su dominio y control. No se puede desconocer que Aristóteles crea una pedagogía de la eficiencia individual y, simultáneamente, de la libertad y de la convivencia social y política (Gadotti, 1998, p. 16). Por este motivo es particularmente importante la instrucción en estos menesteres del control emocional.

En virtud de esta aproximación, Aristóteles es considerado el primer exponente del enfoque fisiológico, del cognitivismo y de la relevancia social de la emoción. Nótese que sobre el incipiente cognitivismo que establece, según Dabrowski (2016), una relación causal entre pensamiento y emoción, Aristóteles señala que

[...] el alma es la unidad de tres partes: racional, sensual y vegetativa. La parte racional presupone la actividad de la razón pasiva y activa, y es responsable de la cognición racional; por otro lado, la parte sensual presupone sentidos, emociones e imaginación. Un principio básico de su ética es que el hombre busca el propósito más elevado, la felicidad, con toda su alma. Significa que las emociones, como parte de ellas, tienen que participar en esa búsqueda. Sin embargo, pertenecen a la parte 
inferior del alma, por lo que están sujetas a la razón... Aristóteles postuló que las emociones son cognitivas, se basan en creencias y evaluaciones.

Cuando un sujeto se encuentra en una situación, sus emociones le informan sobre el significado y el valor de la situación para su vida.

(Dabrowski, 2016, p. 9)

Al plantear, por tanto, que existe una relación causal entre emociones y creencias, Aristóteles deja claro que al establecer la causa, y al cambiarla, cambia también la emoción y, por lo tanto, la conducta. Desde este punto de vista, aconseja que para provocar una emoción en otros es menester cambiar las creencias o el modo de comprender el mundo. En la actualidad, las teorías cognitivas, siguiendo este planteamiento, suponen que la causa de que se produzca una emoción corresponde a una representación mental caracterizada como creencia.

\section{La representación de la emoción como sensación física o la versión objetivista. La versión más transitada: Descartes, la naturaleza pasiva de la emoción y su efecto en la versión James-Lange}

El debate contemporáneo reside, como se ha señalado, en dos posiciones ontológicamente distintas, a saber: que las emociones son meras sensaciones físicas y que son resultado de cogniciones. El problema de su definición, al pasar por la criba del dualismo cartesiano, declara su naturaleza material, perspectiva que William James acogerá para evidenciar el rasgo que permite su lectura objetiva. Y es que tanto Descartes como James se centran en los cambios fisiológicos que tienen lugar cuando se está experimentando la emoción. Descartes sostiene que las emociones son sensaciones que se padecen, que están fuera del control y son irracionales. En Las pasiones del alma en su visión dualista, asegura Descartes (1997) que las emociones son sensaciones, mecanismos físicos, y 
son pasivas, puesto que se padecen, están fuera del control de la voluntad y operan separadas de la razón.

Si bien Aristóteles (1990) ejerce una gran influencia en las aproximaciones a las teorías cognitivas en psicología, es la teoría del sentimiento de Descartes la que influenciará la historia de la filosofía moderna y contemporánea en Las pasiones del alma, desde donde interpreta analíticamente las emociones en su modelo dualista alma-cuerpo. El enfoque cartesiano influencia las primeras tentativas de la psicología en cuanto ciencia experimental - como la del pragmatista William James (James y Lange, 1922), por ejemplo- en virtud de la posibilidad de "medir" los estados internos del sujeto.

Según Descartes (1997), las emociones corresponden al ámbito de lo privado y están desprovistas de elementos cognitivos. Esto significa que no están relacionadas con el pensamiento por pertenecer este a la esfera de lo no corporal, de donde se deduce que las emociones son asuntos puramente corporales. Teniendo esto en cuenta, se puede afirmar que, para él, la emoción es una función y un proceso fisiológico que se manifiesta en la sensibilidad corporal. Debido a esta manifestación sensible, la emoción es sentimiento, en el sentido que se siente corporalmente tal como el frío o el calor. Ninguna emoción es una motivación a la acción, ya que no hay vínculo con el pensamiento y este es el que activa la voluntad, con ello se puede afirmar que no existe conexión entre emoción y conducta, a diferencia de lo que se plantea en las teorías conductistas.

Para Descartes, las pasiones son percepciones porque son algo de lo que las personas son inmediatamente conscientes, son sentimientos porque son recibidas en el alma, del mismo modo que los objetos externos de los sentidos. Kenny (2003) establece que la acción voluntaria para Descartes es lo contrario de la percepción y 
[...] como la percepción es un evento en el alma causado en forma proximal por un movimiento en la glándula pineal, la acción voluntaria es un efecto causado por un movimiento en la glándula pineal que es a su vez causado por un evento en el alma. (p. 6)

Desde este punto de vista, Anthony Kenny, respecto de Descartes, dice que la naturaleza, mediante las sensaciones de placer y dolor, le enseña al alma qué objeto es benéfico o cuál es perjudicial:

Estas sensaciones producen una serie de pasiones que conducen a la acción apropiada para conservar el cuerpo. Así, la sensación de dolor produce la pasión de la tristeza, a la que sigue la pasión del odio por el objeto doloroso y, finalmente, la pasión del deseo de deshacerse de él. O cuando algo se "representa como bueno para nosotros", es decir, cuando se acompaña con una sensación de placer, causa amor en nosotros que conduce a varias formas de comportamiento voluntario apropiado. Las emociones, a diferencia del dolor, tienen objetos: tenemos miedo de las cosas, nos enojamos con las personas, nos avergonzamos de haber hecho tal o cual cosa. Esta característica de las emociones, que a veces se denomina su "intencionalidad". (Kenny, 2003, p. 7)

Descartes entiende las emociones como fenómenos "pasivos", porque precisa distinguirlos de otros fenómenos mentales más "activos" como querer o juzgar. Esta característica de pasividad de las emociones ha sido ampliamente aceptada en la historia de la filosofía, según comentan Calhoun y Solomon (1996).

\section{La versión James-Lange}

Es de advertir que tanto el enfoque cartesiano como la teoría darwinista tienen una fuerte influencia en la interpretación que James hace de las emociones y en su respectiva interpretación organicista-biológica. Es así como se convierte en referente para posteriores estudios relacionados con 
la emoción como mecanismo. James plantea una visión universalista de la emoción mediante la cual sostiene la posibilidad de medir los estados internos del sujeto. Sin embargo, al establecer Descartes que las emociones no se relacionan desde ningún punto de vista con el pensamiento, que su ámbito exclusivo es el cuerpo y son fenómenos pasivos, proporciona el argumento perfecto para la aparición del estudio de la emoción como un fenómeno puramente físico. Es así como William James, haciendo eco del cartesianismo en su enfoque fisiológico, establece que la emoción se manifiesta en la sensibilidad corporal; en este sentido es sentimiento, ya que se siente tal como el frío o el calor ${ }^{1}$.

En esta misma línea de Descartes, William James reconoce que las emociones son simples sensaciones corporales subjetivas, y por lo tanto, establecer su grado de objetividad teniendo en cuenta este aspecto es una empresa imposible. Por ello se queda con su expresión fisiológica, la cual puede aportar "datos" más confiables que la mera narración del sujeto. Sus aportes fundamentales en la comprensión de las emociones son básicamente dos. El primero es la comprensión de la emoción como un cambio fisiológico que puede ser medido. En este sentido, para James es posible contabilizar las diferencias cualitativas y cuantitativas en estos cambios fisiológicos, afirmando que la estimulación eléctrica del córtex desencadena cambios que pueden ser medidos y cuantificados mediante análisis objetivos. El segundo aporte consiste en trasladar el sentimiento, ubicado en el alma aristotélica, al cuerpo, planteando que si existe alguna diferencia en el cambio del sentir, esto obedece al cambio fisiológico implicado, que también es diferente. Es en virtud de estas dos aproximaciones a la emoción que James es considerado como uno de los

\footnotetext{
${ }^{1}$ Se hace la aclaración, porque según ilustra Hillman (1970), el psicoanálisis, por vía de la psiquiatría, establecerá una definición de sentimiento y emoción - que perdura hasta la actualidad-, así como sus diferencias, pero confinando estos dos fenómenos al reino del inconsciente.
}

Hallazgos

ISSN: 1794-3841 | e-ISSN: 2422-409X | DOI: https://doi.org/10.15332/2422409X 
padres de la psicología moderna y de los posteriores desarrollos de las neurociencias.

Las emociones, según su versión, son específicamente sentimientos corporales causados por cambios en las condiciones fisiológicas relacionadas con las funciones autónomas y motoras. Sentir los cambios corporales conduce a percibir o sentir la emoción. Cuando se percibe el peligro, por ejemplo, esta percepción desencadena una colección de respuestas corporales, y la conciencia de estas respuestas es lo que constituye el miedo. Por ejemplo, al encontrarse con una araña, el sentido común dice que eso asusta; por lo tanto, se produce un impulso físico inmediato de respiración rápida, aceleración del pulso, etc., y al sentir estos cambios se produce el miedo. De este modo, según James, el principal componente de las emociones es el aspecto orgánico-fisiológico. No obstante, de acuerdo con lo que señala Kenny (2003), James identifica las emociones no con los procesos corporales propiamente sino con su percepción. De tal manera asegura que las emociones son un tipo especial de percepción, y al respecto explica:

Nuestra forma natural de pensar sobre emociones fuertes es que la percepción mental de algún hecho excita el afecto mental llamado emoción, y que este último estado mental da lugar a la expresión corporal. Mi teoría, por el contrario, es que los cambios corporales siguen directamente la percepción del hecho emocionante, y que nuestra sensación de los mismos cambios a medida que ocurren es la emoción. El sentido común dice perdemos nuestra fortuna, lo sentimos y lloramos; nos encontramos con un oso, nos asustamos y corremos; estamos insultados por un rival, estamos enojados y golpean. La hipótesis aquí defendida dice que este orden de secuencia es incorrecto, que el estado mental no es inducido inmediatamente por el otro, que primero deben interponerse las manifestaciones corporales, y que la afirmación más 
racional es que sentimos pena porque lloramos, enojados porque

golpeamos, temerosos porque temblamos. (James, 1950, vol. II, p. 450)

Es claro que esta línea argumentativa pretende demostrar la naturaleza básica e impulsiva de las emociones determinadas biológicamente, según lo que plantea Darwin.

\section{Darwin: la emoción como función adaptativa}

Esta teoría de la emoción, así como toda la teoría evolutiva de Darwin, encuentra eco en los pragmatistas clásicos, para este caso James y Dewey.

Se ubica en este lugar porque representa un aporte clave en la teoría universalista de las emociones, que es, en cierto modo, lo que pretende demostrar James. En un breve y claro aparte, LeDoux (1999) resume la teoría darwinista de la selección natural así:

Los rasgos que eran útiles para la supervivencia de una especie en un medio determinado se convirtieron a la larga en rasgos propios de la especie. Y, del mismo modo, los rasgos propios de las especies actuales existen porque contribuyeron a la supervivencia de sus antepasados lejanos. Debido a la limitación de las provisiones de alimentos, no todos los individuos que nacen sobreviven hasta llegar a la madurez sexual para reproducirse. Los menos aptos son eliminados, de forma que, con el paso del tiempo, los más aptos se convierten en padres y los hijos heredan esta aptitud. Pero si resulta que el medio cambia, como ocurre constantemente, los rasgos diferentes se vuelven importantes para la supervivencia y acaban siendo seleccionados. Las especies que se adaptan de este modo sobreviven, mientras que las que no se adaptan se extinguen. (LeDoux, 1999, p. 119)

Esta referencia al proceso evolutivo incluye rasgos tanto físicos como mentales y de conducta, y dentro de estas últimas categorías caben las emociones. Según Darwin (2009), estas son funciones como formas de 
respuesta al entorno, como modo no verbal de comunicación entre individuos, cuyo medio son las expresiones faciales, que constituyen una prueba de su universalidad. Por esta razón, las emociones tienen la característica de ser innatas y universales, como se evidencia en el siguiente texto:

Los principales actos de expresión que manifiestan el hombre y otros animales inferiores ahora son innatos o heredados, es decir, el individuo no los ha adquirido [...] el mismo estado mental se expresa en todo el mundo con notable uniformidad; y este hecho es en sí mismo interesante, como evidencia de la similitud cercana en la estructura corporal y la disposición mental de todas las razas de la humanidad. ${ }^{2}$ (Darwin, 2009, pp. 17-18)

De hecho, el argumento de la universalidad de las emociones es utilizado por Darwin para evidenciar su carácter innato.

Según Calhoun y Solomon (1996), Darwin intenta explicar el origen de la conducta (expresión) emocional con arreglo a su utilidad para la supervivencia. En este sentido, plantea tres principios que explican la razón de ser de estas conductas. El primer principio obedece a una simple reacción o impulso para evitar ser dañado o morir, el cual se va convirtiendo en un hábito que se puede transmitir genéticamente - adoptando la ya desacreditada explicación lamarckiana-, y es así como deviene innata. El segundo principio, el de la antítesis, "opera como contraposición de conductas útiles relacionadas con emociones opuestas" (Calhoun y Solomon, 1996, p. 19). El tercer principio, el de la acción directa, establece que ciertas conductas son derivadas de la excitación corporal en una experiencia emocional inicial. Curiosamente, para Darwin, la conducta es mera expresión de la emoción, a diferencia de la acepción

2 Traducción propia.

Hallazgos

ISSN: 1794-3841 | e-ISSN: 2422-409X | DOI: https://doi.org/10.15332/2422409X

Vol. 18 N.0 36| julio-diciembre del 2021 
de conducta proveniente del conductismo. De acuerdo con Calhoun y Solomon (1996), para Darwin, la emoción "es un fenómeno preciso que causa la conducta emocional [...] aparentemente estaba de acuerdo con los teóricos de la sensación y con los fisiológicos en que las emociones son experiencias internas y privadas" (p. 20).

La importancia de esta interpretación de las emociones consiste en la contribución de Darwin a su naturalización, en la medida en que las considera como sustratos fundamentales de la vida instintiva y como rasgos universales adaptativos esenciales para la supervivencia.

Teniendo en cuenta esta última interpretación como influencia del pensamiento de James sobre las emociones, su línea argumentativa pretende sostener que la razón es una función secundaria, lenta, en tanto que las emociones son enteramente biológicas, impulsos rápidos, no racionales. Su argumentación apela al modelo biológico darwinista de supervivencia, según el cual se establece una intrincada conexión entre emoción y acción, donde una emoción es la experiencia de sentir el cuerpo que se prepara para actuar. Esta perspectiva se basa en la teoría darwinista para la que los organismos requieren actuar de manera rápida, lo que garantizaría la supervivencia y la adaptación al entorno. Hacer pasar las respuestas del organismo por el pensamiento o por estados mentales no garantizaría la función adaptativa de la emoción.

Para James, esta teoría se sostiene por sí misma, puesto que si se eliminan los síntomas corporales, la emoción desaparecería porque no es posible experimentar, por ejemplo, el miedo solo mentalmente sin la aparición de manifestaciones corporales evidentes. Por último, y en la misma línea argumentativa, James sostiene que es posible controlar las emociones, controlando estados corporales, de tal modo que se puede reducir el ritmo cardiaco mediante ejercicios de respiración, lo que conduce a reducir los síntomas corporales, y por tanto, la emoción desaparece. 
Ahora bien, algunas de las objeciones a la teoría de James provienen de Schachter y Singer y de la teoría de Cannon-Bard. Esta última es la que abre la puerta al cognitivismo, tan popular actualmente.

\section{Schachter y Singer: la expresión fisiológica de la emoción}

Schachter y Singer (1962) realizaron el experimento más conocido relacionado con las emociones.

Estos experimentos manipularon de forma independiente los determinantes fisiológicos y situacionales del estado emocional. Se demostró que ni las explicaciones fisiológicas ni psicológicas por sí solas podían explicar los hechos experimentales y se propuso una visión interactiva de la emoción. (p. 379)

Este experimento tiene como propósito la identificación de la emoción con base en su expresión fisiológica. Concluye que algunos estados fisiológicos pueden ser nombrados como correlativos a una determinada emoción. Sin embargo, también concluye que no es posible distinguir una emoción de otra solamente en referencia a la manifestación fisiológica. Este experimento es muy conocido por cuanto muestra que no es posible identificar la manifestación fisiológica de las emociones, aunque difieran en su causa. De manera muy sucinta el experimento consiste en administrar adrenalina e informar a un grupo de personas, mientras que a otro grupo se le administra pero no se le informa. El resultado es que los informados presentan síntomas de ira, mientras que a quienes no se informa interpretan los efectos fisiológicos de la adrenalina como emociones positivas. Esto permite concluir que la diferencia entre las emociones no es fisiológica sino cognitiva.

Así mismo, este estudio demuestra que la percepción de procesos corporales similares permite interpretarlos como emociones contrarias según el contexto que se ofrece para ello. Al parecer, la respuesta corporal 
no es tan importante como la interpretación de dicha respuesta. En este sentido, el significado y el modo de interpretar según la información es lo que determina la respuesta emocional.

Vale decir que los resultados de este estudio abren la puerta para preguntarse, en primer lugar, por la articulación entre el contexto de significación y las respuestas emocionales, lo que permite pensar que las emociones en las distintas culturas cambian según la estructura de significación presente en cada una de ellas. Así mismo, abren la posibilidad para pensar que las emociones no son meras expresiones fisiológicas, sino más bien procesos altamente sofisticados, por lo que se puede considerar la idea de que las emociones sean intencionales por cuanto la intencionalidad consiste en la capacidad para representar los procesos corporales como tendiendo hacia algo. Esta es otra vía de estudio que se abre con los resultados de Schachter y Singer.

\section{Cannon-Bard}

Según Lagier (2009), al intentar confirmar la teoría James-Lange, Cannon se encuentra con interesantes resultados que contrariamente la rebaten y dejan abierta la puerta para una explicación, desde la cual surge la teoría psicológica de Cannon-Bard. Sostiene Cannon que los mismos cambios fisiológicos se observan en estados emocionales muy diferentes y en estados meramente corporales como la fiebre, el frío, etc., es decir, muchas emociones se asocian a las mismas expresiones corporales, lo que dificulta identificar cuál emoción corresponde claramente a cada cambio corporal. Por ejemplo, la ira y el miedo tienen la misma manifestación corporal, a saber: sudoración, palpitaciones y respiración acelerada. Al parecer, asegura Cannon, las emociones difieren por su causa y no por su manifestación física, por eso esta va un paso atrás a la causa, es decir a la valoración cognitiva. Esta explicación centra su interpretación en los 
procesos mentales. En este sentido, es una explicación cognitiva en la medida en que entiende el proceso emocional como la percepción de un estímulo que es enviado al cerebro y seguidamente se hace una valoración cognitiva para producir una respuesta corporal.

Aquí se abre la puerta para al menos dos objeciones: una de orden antropológico y otra de carácter fenomenológico. Según la primera, se evidencia la existencia de una variabilidad cultural desde la cual las emociones se educan, experimentan e interpretan con base en la cultura. Según la objeción fenomenológica, las emociones exhiben intencionalidad y son significativas, no son simples respuestas corporales; involucran el pensamiento por cuanto las emociones pueden tener causas. Son un "hacia algo".

No todas las emociones son intencionales, pero la mayoría tienen una naturaleza intencional.

\section{La versión cognitivista de la emoción: "el giro cognitivo"}

Este apartado tiene por objeto mostrar de manera sucinta el panorama cognitivista de las emociones y sus desarrollos. Vale decir que las distintas teorías se basan en el origen mental de la emoción. Si bien todas las teorías están de acuerdo en que conocer, interpretar y evaluar constituyen el centro de la emoción, difieren de manera taxativa en su definición del sentido de "cognición”. Este giro cognitivista se inspira en Aristóteles, quien, como ya se ha visto, plantea que las pasiones son las causantes de que los hombres se hagan volubles y cambien sus juicios, lo que significa que cambiar las emociones supone cambiar las convicciones.

Las teorías cognitivas, siguiendo este planteamiento, suponen que la causa de que se produzca una emoción corresponde a una representación mental caracterizada como creencia. La diferencia de emociones para estas teorías 
se encuentra en las distintas creencias que se abrigan sobre el mundo. Por lo anterior, se puede deducir que al ser la creencia la causa de la emoción, entonces las creencias la anteceden, y puesto que cada individuo se emociona de manera distinta en virtud de sus creencias, se puede afirmar que las emociones son radicalmente íntimas y subjetivas. Este problema de la naturaleza subjetiva de la emoción se tiende a pasar por alto, ya que el objetivo es buscar patrones explicativos, modelos y teorías, por lo que no caben singularidades ni particularidades.

De acuerdo con Dabrowski (2016), los cognitivistas afirman, siguiendo una de las líneas de análisis de Aristóteles sobre las emociones, que estas se encuentran estrechamente relacionadas con pensamientos, creencias o juicios.

Los partidarios de las teorías cognitivas asumen [...] que las emociones requieren conceptos y creencias, y también que existe una clara distinción entre los ámbitos somático y cognitivo. Este último se localiza de alguna manera, en algún ámbito por encima de los cambios en el cuerpo. Esos cambios no son relevantes para el surgimiento de la emoción. Los defensores de varias teorías cognitivas subrayan propiedades de las emociones como: intencionalidad, carácter cognitivo, conceptualización, dependencia de pensamientos, creencias o juicios, capacidad para evaluar eventos, etc. ${ }^{3}$ (Dabrowski, 2016 p. 15)

El giro cognitivo tiene lugar en el siglo XX y es originado por los experimentos de Cannon-Bard. Estas teorías son sugestivas por cuanto al basar las emociones en contextos epistemológicos de interpretación del mundo, resuelven los problemas creados por Darwin-James-Lange al hablar de universalidad de la emoción. El cognitivismo prospera en psicología con los textos de Magda Arnold y en filosofía con Anthony

3 Traducción propia.

Hallazgos

ISSN: 1794-3841 | e-ISSN: 2422-409X | DOI: https://doi.org/10.15332/2422409X

Vol. 18 N.0 36| julio-diciembre del 2021 
Kenny y Errol Bedford. Ambas se construyen sobre una de las líneas de análisis de Aristóteles. Así, Arnold (1970) sostiene que aunque el aspecto fisiológico es importante, el modo como es interpretado por el sujeto es un importante aspecto. Para Arnold, hay problemas relacionados con las emociones que continúan sin ser resueltos: el problema de cómo se relacionan la emoción y la acción, el de cómo aparece la emoción, cómo se producen los cambios fisiológicos y cuál es la diferencia entre la emoción que impulsa a la acción y aquella que interfiere con ella (Arnold, 1970, p. 172). Para esta autora la emoción es una acción-tendencia sentida subjetiva como un sentimiento emocional característico que se manifiesta en función del patrón fisiológico (objetivo). Sin embargo, este abordaje hoy en día se critica porque se cuestiona si efectivamente los patrones fisiológicos pueden definir las emociones específicas, y en este sentido se pone en tela de juicio si el mismo patrón no se manifiesta con distintas emociones.

Errol Bedford (1956-1957) cuestiona "la función de las proposiciones respecto de las emociones y los criterios para su validez" (p. 281). De este modo, rebate la versión aceptada sobre la naturaleza de la emoción y la puesta en duda de que una emoción sea un sentimiento. Por su parte, Anthony Kenny considera que existen condiciones bajo las cuales se aprende el significado de una emoción, mediante un ejemplo: el del miedo, entiende que

[e]l concepto de cada emoción está ligado a conceptos no emocionales de tres maneras. Por ejemplo, el concepto de miedo descansa sobre tres pilares: a) las circunstancias “temibles”, b) los síntomas del miedo, c) la acción emprendida a fin de eludir lo que se teme.

Así como la expresión verbal del miedo debe ser aprendida en el contexto de dichos factores, de la misma manera únicamente puede ser comprendida en el contexto de una u otra forma de los mismos. En el 
caso estándar, que es al tiempo el paradigma y el más fácilmente inteligible, los tres factores se hallarán presentes. El león devorador de hombres se aproxima rugiendo; el indefenso plantador, chilla, palidece, y se da a la huida. Su posterior testimonio "estaba aterrorizado", es tan plenamente inteligible como lo puedan ser tal tipo de testimonios. Sin embargo, la expresión verbal del miedo continúa siendo inteligible cuando uno, o incluso dos de estos factores está ausente, pero el tercero permanece. (Kenny, 1963, p. 67)

Una de las más conocidas teorías cognitivas asegura que las emociones son pensamientos o juicios. Se trae este ejemplo para mostrar la relación con James y seguir avanzando en lo que se ha propuesto, que no es precisamente ahondar en los debates contemporáneos. En esta línea de argumentación de la identificación entre juicios y emociones se encuentran Nussbaum y Solomon. Según esta versión, para experimentar una emoción no se requieren sensaciones corporales: con el pensamiento o la creencia es suficiente. Nótese que esta es la inversión de la teoría de James, porque en el caso de Nussbum se considera que, al experimentar una emoción intensa, solo se requiere un pensamiento que la provoque, no necesariamente un elemento corporal. En este sentido, para Nussbaum (2008), las emociones son "formas de juicio" (p. 44) con base en la caracterización que elabora, y aunque se presenten de manera física, las emociones se vinculan con el pensamiento por

[s]u apremio y su calor: su inclinación a apoderarse de la personalidad e impulsarla a la acción con una fuerza arrolladora; su relación con vínculos importantes, con respecto a los cuales la persona define su vida; la sensación de pasividad ante ellas; su aparente relación de confrontación con "la racionalidad", en el sentido de un cálculo frío o de un análisis del tipo coste-beneficio; el estrecho vínculo entre ellas, pues la esperanza alterna de modo titubeante con el temor, o un solo acontecimiento transforma la esperanza en aflicción, o la aflicción en 
busca de una causa, se expresa en forma de ira, a la vez que todas ellas puedan ser el vehículo de un amor subyacente. (p. 44)

En la exhaustiva clasificación realizada por Vendrell (2009), se muestra claramente el debate contemporáneo sobre la emoción. No es necesario detenerse en el debate, solo se incluye a fin de mostrar el ámbito en el cual orbitan hoy las preguntas por la emoción en filosofía. En este escenario aparecen dos posiciones: quienes afirman que las emociones tienen una función cognitiva y quienes establecen que las emociones se basan en actos de pensamiento.

Según la primera posición, las emociones aportan información sobre el mundo. Dentro de esta perspectiva se pueden identificar a su vez dos posiciones. Una es de carácter reduccionista, que afirma que la función cognitiva de las emociones es posible simplemente porque estas son cogniciones, es decir, son un tipo particular de juicios: juicios valorativos o evaluaciones de objetos externos hacia los cuales se dirige (intencionalidad). La otra es de tipo no reduccionista; establece que el aspecto cognitivo de la emoción se refiere a que esta es una percepción que aprehende cualidades axiológicas de los objetos. Estas valoraciones pueden ser constituidas socialmente o también pueden ocurrir por ser proyecciones de los estados afectivos del individuo o, según quienes sostienen el realismo ontológico, los valores tienen una existencia independiente de los individuos y el sentir que la emoción corresponde con una cierta capacidad de acceso a este mundo de valores.

De acuerdo con la segunda postura, que las entiende como actos de pensamiento, hay dos posiciones marcadas, a saber: la reduccionista -Taylor (1985) y Green (1992) - , la cual establece que las únicas bases cognitivas de la emoción son los juicios que permiten individualizarlas; y la no reduccionista de Elster (2002), quien considera que las bases 
cognitivas de la emoción son de diferentes tipos: suposiciones, juicios, recuerdos, fantasías, percepciones, etc.

La mayoría de las teorías sobre la emoción, tanto psicológicas como filosóficas contemporáneas, caracterizan las emociones principalmente respecto a las cogniciones a las cuales se vinculan. Pero, como se ha dicho, hay múltiples formas de proponer interpretaciones de estas cogniciones. Mientras que los teóricos de la evolución generalmente entienden que los procesos cognitivos subyacentes a la emoción puedan ser conscientes o inconscientes, y pueden involucrar contenido proposicional o no proposicional, los cognitivistas típicos afirman que las emociones involucran actitudes proposicionales. Muchas emociones se especifican en función de proposiciones: uno no puede estar enojado con alguien a menos que uno crea que esa persona es culpable de alguna ofensa; uno no puede tener envidia a menos que uno crea que alguien más tiene algo bueno en su posesión. Algunos defensores del cognitivismo universalizan esta característica y sostienen que cualquier emoción debe involucrar algún tipo de actitud dirigida a una proposición.

\section{El surgimiento del "cerebrocentrismo"o de por qué la perspectiva fisiológica-objetivista ha tenido tanto eco en el estudio sobre las emociones}

Habiendo considerado las perspectivas desde las cuales se aborda el problema de las emociones, y teniendo en cuenta en torno a qué aspectos orbitan los debates contemporáneos, se puede ver que hoy ha tomado mucha fuerza su estudio con base en el uso de tecnologías que permiten reconocer zonas del cerebro asociadas a ciertas emociones. Las neurociencias se han constituido en el relato más aceptado; incluso los estudios psicológicos están basados en el dato científico proveniente de estas demostraciones. ¿¿De dónde viene este particular entusiasmo por este unívoco relato? Se aventurará aquí un esbozo de respuesta, no sin antes 
advertir que a pesar de la inclinación por esta versión de la emoción, se han establecido diálogos con las ciencias sociales para poder tener una versión más completa de este interesante fenómeno.

En el siglo XVIII, como resultado de la herencia cartesiana, se fortalece la representación que establece que las emociones son fenómenos que se producen en la mente y el cuerpo. Se desplaza el alma como ámbito de las pasiones o la mente sin cuerpo como su origen, y en su lugar se elige emplazando las emociones en un espacio concreto: el cerebro, más precisamente: el cerebro límbico, que corresponde al sustrato orgánico o fisiológico de lo que hoy denominamos mente, según la psicología filosófica. Desde el siglo XIX, en el momento en que la ciencia positiva se robustece y deviene hegemónica, este abordaje orgánico o fisiológico conduce a la cerebrocentrismo ${ }^{4}$ de todas las funciones relacionadas con el sujeto, el cual da origen a la plena representación psicofisiológica. Según esta representación, las emociones son definidas como entidades orgánicas (fenómenos psicofisiológicos) de la mente que ocurren específicamente en la corteza cerebral y el hipotálamo, de manera que la emoción adquiere concreción física. Esta ubicación precisa de las emociones en un lugar concreto contribuye en gran medida a instaurar su universalización. Sin embargo, se podría preguntar con Papanicolau (2004) "por qué el cerebro es una mejora sobre la mente como principio último explicatorio" (p. 29). Ante esto se responde con toda certeza que en virtud de la materialidad del cerebro es posible seguir las metodologías de

\footnotetext{
4 "'cerebrocentrismo'. Consiste en una fascinación casi mística y total devoción hacia el cerebro como la única fuente de todo fenómeno que merece consideración en psicología. La forma moderna de este sesgo es, al menos parcialmente, debida al incremento del refinamiento de las técnicas para la manipulación y observación directa del cerebro, y debido a la consecuente apreciación de la enorme complejidad de este órgano y el alcance y sutileza de sus efectos sobre el comportamiento. De este modo, casi reflexivamente, todo fenómeno psicológico es atribuido al cerebro y la mayoría de los esfuerzos se dedican a la búsqueda de las causas de todos los fenómenos en sus varios procesos y retrocesos" (Papanicolaou, 2004, pp. 28-29).
}

Hallazgos

ISSN: 1794-3841 | e-ISSN: 2422-409X | DOI: https://doi.org/10.15332/2422409X

Vol. 18 N.o 36| julio-diciembre del 2021 
estudio de la ciencia en cuanto se le puede aplicar la observación y la manipulación experimental. En este sentido, se coincide con este autor en la siguiente tesis:

Sea como sea, tanto si la "mente" de la psicología filosófica o el "cerebro" de la psicología científica son utilizados como principios explicatorios, la emoción sigue explicándose con solipsismos y profundas tautologías: las causas de la experiencia emocional están en la mente o en el cerebro, la experiencia emocional es el darse cuenta de los procesos cerebrales, cualidades de la mente, o procesos cognitivos. (Papanicolau, 2004, pp. 29-30)

\section{La consecuencia natural: la representación de la emoción desde las neurociencias}

El aporte de William James y Carl Lange es fundamental para que la psicofisiología avance y se pueda determinar con claridad el estatuto biológico y la función evolutiva de las emociones. Las neurociencias se ocupan de investigar el funcionamiento del cerebro y el sistema neuronal propiamente, de modo que se permita la descripción y el reconocimiento de las bases cerebrales del comportamiento. Por ser un campo de estudio tan amplio, las neurociencias se subdividen en neurociencia cognitiva, neurociencia social y neurociencia afectiva. Esta última se ocupa de reconocer los procesos neuroquímicos presentes en la emergencia de las emociones. Por otro lado, la neurociencia (Mora y Sanguinetti, 1994) tiene como objeto de estudio el sistema nervioso, su influjo en los procesos cognitivos y en el comportamiento humano.

Damasio (1994), desde las neurociencias, plantea la teoría del marcador somático. Según esta, existe un vínculo entre los mecanismos emocionales y las decisiones que determinan la acción a largo plazo, lo que pone en tela de juicio que las emociones sean meros impulsos que orientan la acción inmediata. En este sentido, señala Damasio que mediante complejos 
procesos cognitivos, emocionales y neuronales, las emociones se convierten en señales somáticas, las cuales, por medio de regulaciones de tipo cognitivo, pueden producir modificaciones tanto somáticas como de significación en los estados emocionales.

Nótese que Damasio (2009) entiende las emociones como funciones que “apuntan directamente a la regulación vital a fin de evitar los peligros o ayudar al organismo a sacar partido de una oportunidad, o indirectamente al facilitar las relaciones sociales" (p. 43). Desde esta perspectiva, la emoción es un proceso cerebral no distinto cualitativamente de cualquier otro proceso cognitivo. Por ser las emociones mecanismos cerebrales primitivos e instintivos respecto a la evolución, se ponen en marcha de manera inmediata (al hacer el mismo estudio con animales, aparecen los mismos mecanismos). Esto lo lleva a plantear que la conducta observable tiene un correlato en el cerebro, y al ponerse en marcha como mecanismos inconscientes e inmediatos sin el concurso del pensamiento, se concluye que los procesos cognitivos están sometidos, alterados modificados o eliminados por las emociones, y no al contrario.

$\mathrm{Al}$ respecto, señala:

[...] tenemos emociones primero y sentimientos después porque la evolución dio primero las emociones y después los sentimientos. Las emociones están constituidas a base de reacciones simples que promueven sin dificultad la supervivencia de un organismo, y de este modo pudieron persistir fácilmente en la evolución. (Damasio, 2009, p. 34)

Por último, Damasio, desde la neurociencia, plantea que al ser la emoción un rasgo resultado de la evolución, su función está vinculada con la supervivencia de la especie, en la medida en que se convierte en un elemento que previene del peligro de lesión o muerte. Su eventual 
aparición, con los consiguientes signos físicos, está orientada a la conservación de la vida.

Desde el punto de vista práctico, comprender la biología de las emociones y el hecho de que el valor de cada emoción difiera tanto en nuestro ambiente humano actual, ofrece considerables oportunidades para entender el comportamiento humano. Podemos aprender, por ejemplo, que algunas emociones son consejeras terribles y considerar de qué manera podemos suprimirlas o reducir las consecuencias de su consejo. Pienso, por ejemplo, que las emociones que conducen a prejuicios raciales y culturales se basan en parte en el despliegue automático de emociones sociales destinadas, desde el punto de vista evolutivo, a detectar la diferencia en los demás, porque dicha diferencia puede señalar riesgo o peligro, y promover la retirada o la agresión. Este tipo de reacción probablemente consiguió objetivos útiles en una sociedad tribal, pero ya no es eficaz, y mucho menos apropiada, en la nuestra. Podemos darnos cuenta de que nuestro cerebro porta todavía la maquinaria para reaccionar de la manera en que lo hizo, en un contexto muy diferente, hace muchísimo tiempo. Y podemos aprender a desechar tales reacciones y persuadir a otros para que hagan lo mismo. (Damasio, 2009, p. 44)

\section{La representación de la emoción como un fenómeno social: las ciencias sociales, el aspecto expresivo de la emoción}

Una de las aportaciones más importantes de las ciencias sociales ha consistido en señalar que las emociones son fenómenos situados, tanto histórica como culturalmente. De aquí se resalta su emergencia como resultado de estructuras sociales que definen las narrativas, los léxicos y las manifestaciones de este fenómeno. La importancia radical de esta perspectiva es la crítica a la idea de que las emociones sean un fenómeno universal. 
Estas interpretaciones sostienen que las emociones no son simple y únicamente biológicas, sino que existen razones de orden social que las configuran para que se expresen en el rostro y en el cuerpo de una cierta manera, y que haya otros que comparten la misma forma de interpretarlas y sean capaces de leerlas. Como la palabra emoción se deriva del latín emovere, que significa 'mudarse', se colige que el significado mismo del término sugiere que las emociones consisten en poner de manifiesto a los observadores el estado interno del individuo, con lo cual evidencia la naturaleza social del fenómeno. Aunque es claro que las emociones se experimentan en un ámbito privado, también es cierto que se reflejan mediante expresiones faciales o corporales.

El mismo hecho de que las emociones tiendan a expresarse significa que pueden tener funciones y consecuencias sociales además de sus efectos intrapersonales ampliamente estudiados y aceptados. En este sentido, las emociones pueden ser pensadas como construcciones, conexiones y modos de compromiso con el entorno social.

¿Desde cuál campo es posible estudiar la emoción en su dimensión social? Hoy las emociones son un campo de investigación abordado por la historia, la sociología, la antropología, la psicología, precisamente porque es claro que estas no son un fenómeno exclusivamente psicológico o fisiológico, y aunque se expresen con el cuerpo constituyen un fenómeno complejo. Entenderlas solo como un mecanismo fisiológico implica despojarlas de su riqueza fenomenológica expresiva, del mismo modo que entenderlas solo como construcción social implica desconocer su dimensión fisiológica o psicológica privada. Cada una de estas aproximaciones ofrece elementos esclarecedores de este fenómeno, en sus aspectos tanto individuales como sociales.

Existen hoy en día muchos estudios sobre las funciones sociales y las consecuencias de las representaciones emocionales. Los investigadores 
sociales examinan el modo como las expresiones emocionales moldean las relaciones cercanas, influyen en los procesos grupales, regulan y afectan el comportamiento de la colectividad. Nótese que el abordaje de las ciencias sociales se ubica en otro escenario de su comprensión: la mediación de la expresión en la intersubjetividad.

Olivia López (2016), desde los estudios de las ciencias sociales de las emociones Renisce, plantea que en la psicología las emociones han sido abordadas a partir de lo que se designa como una "lógica de patologización, porque se conciben como fenómenos psicofisiológicos causantes o a veces resultantes de las enfermedades mentales”. En las investigaciones de López se expone claramente que las emociones constituyen una dimensión sociocultural a partir de la cual es posible leer horizontes de sentido, ideologías, valoraciones e interpretaciones del mundo que hacen posible comprender el modo como la experiencia de los individuos transforma la acción social, y de cómo las estructuras sociales dan forma a modos de emocionar situados social, cultural e históricamente. Así mismo, en otras disciplinas de las ciencias sociales, el tema de las emociones ha sido abordado en la historia por Norbert Elias, Johan Huizinga y Ute Frevert; en sociología, por Thomas Scheff, Theodore Kemper y Arlie Hochschild, y en antropología, por David Le Breton (1998), Michele Rosaldo (1989) y Catherine Lutz (1998).

De acuerdo con Ute Frevert (2011), las neurociencias no solo han logrado ubicar las emociones con "precisión", sino también han desarrollado un conocimiento científico, además de estrategias tecnológicas para identificarlas, clasificarlas, estudiarlas, aprenderlas y manejarlas adecuadamente.

La moda actual sobre la "inteligencia emocional" ofrece solo un ejemplo reciente de una larga tradición de libros de consejos y técnicas de capacitación dirigidas a mejorar el capital social y la eficiencia económica 
de una persona. Al mismo tiempo, la cultura de "lo terapéutico" que surgió en el siglo XX ha reforzado la introspección y la conciencia emocional como medios cruciales para la salud psíquica individual y el equilibrio. (Frevert, 2011, pp. 16-17)

Por otro lado, Frevert (2011), sobre la psicología, dice que es una disciplina que se fue alejando poco a poco de la filosofía y la metafísica para irrumpir en todo tipo de ámbitos:

[...] desde las relaciones industriales hasta la publicidad comercial, desde la terapia individual hasta la comunicación política. En su estela, las emociones ganaron atención y permearon el discurso público, así como las conversaciones privadas entre los amantes, los amigos y la familia. (p. 18)

Las emociones refuerzan narrativas que le otorgan preeminencia al individuo, como si participaran en las lógicas propias del neoliberalismo económico.

En la discusión del desarrollo emocional, dos modelos prototípicos de emoción se han utilizado para contrastar las diversas teorías: el biológico universal y el sociocultural relativizado. Hochschild (1983) discutió el modelo biológico, que se centra principalmente en los aspectos constantes, universalizables, de la expresión más que en las diferencias individuales de expresión que pueden requerir interpretaciones de socialización situada histórica y geográficamente. Por eso rechaza de plano la interpretación organicista-biológica de Darwin y James, y escoge la aproximación interaccionista-situacional de Dewey. A partir de esta propone que aunque la biología es un primer sustrato básico, es necesario añadir elementos de orden social. Es así como lo social está en una permanente interacción con lo emocional en el proceso de la experiencia del individuo. Su principal preocupación consiste en mostrar la manera como opera el ser humano en 
el ámbito emocional, y no tanto en encontrar la causa social de las emociones en los individuos. Hochschild junto con Scheff y Kemper son los primeros sociólogos en preguntarse por las emociones y su relación con las estructuras sociales en la década de los ochenta. Proponen conceptos útiles para explicar la función social de las emociones y contribuir, según señala Bericat (2000), a descubrir nuevos fenómenos sociales.

Kemper, por ejemplo, según su teoría sociorrelacional, aborda el problema de las emociones, intentando desentrañar el modo como las relaciones se definen en virtud del poder. Este, según Kemper (2011), “cubre ampliamente [lo que él llama] estado y el último poder” (p. 13), y el estatus que se otorga de manera voluntaria a otro, "por la posición de los actores con respecto a su cumplimiento de los deseos, necesidades e intereses de otros actores" (p. 13). Estas dos dimensiones relacionales constituyen las formas básicas de las relaciones sociales, y aunque Kemper no niega el origen biológico de las emociones, reconoce que la dimensión social las "desencadena" de una cierta manera. Así, considera que el poder demarca relaciones mediadas por la coerción y la amenaza, y el estatus define relaciones de aprobación, aprecio, amor, etc.

En este mismo horizonte sociológico, Scheff considera que el imperativo emocional en los seres humanos está determinado por mantener los vínculos sociales con los otros, vínculos a los cuales él denomina seguros, determinados por el grado de cercanía o distancia entre individuos, y que son la fuerza que une a una sociedad,

[...] esta fuerza implica un equilibrio entre cercanía y distancia, lo que Bowen (1978; Bowen y Kerr, 1988) ha llamado “diferenciación”. (Bowen, un psiquiatra, es uno de los creadores de la teoría del sistema familiar). La diferenciación óptima define un vínculo social intacto, un vínculo que equilibra las necesidades del individuo y las necesidades del grupo. 
Implica poder mantener lazos con otros que son diferentes de uno mismo. (Scheff, 1990, p. 4)

Por su parte, la antropología entiende que las emociones son más creación de la cultura que rasgos de la naturaleza. En esa medida, son productos culturales simbólicos con funciones ideológicas que socializan sus contenidos mediante el lenguaje. En este sentido, Le Breton explora la dinámica de la emoción en diferentes culturas, señalando diferencias y comparando similitudes. Para él, los factores de socialización de la emoción son considerados cuando surgen preguntas alrededor del estímulo y la expresión de las emociones. Aunque las emociones son respuestas biológicas, funciones orgánicas, están sujetas a la socialización, es decir, su expresión e interpretación están influenciadas por relaciones sociales, de modo que se integran dentro de las estructuras y normas propias de una cultura. Según Le Breton (1998), las emociones corresponden a la intimidad psicológica de los individuos, pero son socialmente configuradas en cuanto a significación e interpretación, en una serie de símbolos corporales. Señala que la emoción constituye un fenómeno que relaciona al individuo con el mundo e implica una forma de interacción que se aprende mediante la experiencia de dar sentido afectivo a la realidad. $\mathrm{Al}$ respecto, dice que las emociones "son pensamientos en acto apoyadas en un sistema de sentidos y valores" (p. 11):

[...] arraigadas en una cultura afectiva, se inscriben a continuación en un lenguaje de gestos y mímicas en principio reconocible (a menos que el individuo disimule su estado afectivo) por quienes comparten sus raíces sociales. La cultura afectiva brinda esquemas de experiencia y acción sobre los cuales el individuo borda su conducta según su historia personal, su estilo y, sobre todo, su evaluación de la situación. La emoción sentida traduce la significación dada por el individuo a las circunstancias que repercuten en él. Es una actividad de conocimiento, 
una construcción social y cultural que se convierte en un hecho personal a través del estilo propio del individuo. (p. 11)

Las emociones, según su enfoque, implican múltiples contenidos culturales específicos de colectividades, etnias y comunidades, las cuales expresan sus representaciones del mundo; "expresan mediante creencias, costumbres y ritos destinadas a las situaciones más relevantes de la comunidad (los nacimientos, la muerte, la enfermedad, etc.), estas emociones son proposiciones que la cultura le otorga al sujeto" (Le Breton, 1998, p. 10). Esto implica que cada colectividad experimenta los acontecimientos de una cierta manera, y en consecuencia expresan dichas representaciones afectivamente

a través de repertorios culturales diferenciados que a veces se parecen, pero no son idénticos. Cada término del léxico afectivo de una sociedad o un grupo social debe ponerse en relación con el contexto local de sus puestas en juego concretas. (Le Breton, 1998, p. 10).

\section{La teoría de la emoción de Dewey: la "otra" vertiente del pragmatismo, la interpretación interaccionista-situacional o la vuelta a la unidad cuerpo-mente}

La teoría de la emoción de John Dewey5, se incrusta en su proyecto de superar la dualidad cuerpo-mente, como la planteó Aristóteles, donde desde su concepto del arco reflejo en psicología4 (EW 5, p. 96-109), la

\footnotetext{
${ }^{5}$ Se toman como referencia las obras completas, Complete Works, editadas por Jo Ann Boydston de la Universidad de Chicago, las cuales se agruparon por año de publicación: The Early Works: 1882-1898 (5 vols.); The Middle Works:1899-1924 (15 vols.); The Later Works 1925-1953 (17 vols.); Southern Illinois University Press (1981-1986).

${ }^{4}$ Dewey pone en tela de juicio la creencia tradicional en psicología, según la cual "el estímulo y la respuesta son dos entidades mentales distintas que ocurren en una conjunción mecánica de procesos desagregados, en los cuales la sensación ocurre primero, la idea la sigue y luego tiene lugar la acción (EW 5, p. 97). [Para él, debe existir] una 'coordinación' previa a dicho estímulo [...], el estímulo es resultado de una coordinación y el acto es un aprendizaje apropiado por un mecanismo de significación que le da sentido" (Patiño, 2011, p. 71).
}

Hallazgos

ISSN: 1794-3841 | e-ISSN: 2422-409X | DOI: https://doi.org/10.15332/2422409X

Vol. 18 N.0 36| julio-diciembre del 2021 
percepción es una red compleja de impresiones significativas. Aquí "el cuerpo 'experimenta' el mundo, como una unidad que corresponde a todos los órganos de los sentidos, a las emociones y a las acciones, de suerte que lo que experimenta no son los sentidos, sino nosotros" (Patiño, 2011, p. 70). Por eso estímulo, experiencia, significación y acción no están desarticulados, ni son sucesivos en el tiempo; ocurren de manera coordinada al mismo tiempo.

Esta versión de las emociones difiere radicalmente de la teoría de James, por cuanto no intenta universalizar la emoción, tampoco aislarla de todo el proceso, pero sobre todo porque tiene en cuenta el marco de significación que hace posible la emergencia de la emoción, y es en este sentido que su teoría solo ha tenido eco en las ciencias sociales. Como lo muestro en Patiño (2014), esta teoría se explica en Art as Experience (LW 10), Theory of Emotion (EW 4, p. 152-188). En esta última las emociones se vinculan con la expresión y la acción creativa, transformadora de las situaciones. En sus escritos, Dewey articula las emociones con todo el proceso creativo y expresivo. Distingue claramente entre una emoción y un impulso reflejo, el cual constituye una respuesta inmediata o automática, como saltar de un susto (Dewey, LW 10, p. 49). Plantea que para que un acto sea propiamente emocional debe ser activado por una situación que se interprete como fuente de tensión. Esta tensión provee la energía (emoción) para resolver la situación 'desequilibrante', lo que significa que la emoción es una dinamizadora de la acción, la cual opera como mediación entre la intención tradicionalmente entendida como un evento 'interno' o 'privado' y la acción, interpretada como 'externa'; así las cosas, el carácter "privado" de la emoción no es tal, ... [por cuanto se hallan] "conectadas a eventos... que son deseados o rechazados" (Dewey, LW 10, pp. 48-49) y en este sentido, demandan resolución en la acción en el mundo concreto. Esto supone un marco de referencia interpretativo que 
permita desear o rechazar. Ahora bien, un impulso reflejo puede pasar a ser emoción una vez que entra el pensamiento en juego, es decir, "cuando se piensa que existe un objeto amenazador del que se debe escapar o con el que se debe lidiar [...] la emoción es la fuerza que desencadena y consolida toda la experiencia" (Dewey, LW 10, p. 49).

Dewey explica las emociones a partir de su modo de expresión, puesto que aunque el reflejo tanto del animal como el del individuo sea idéntico, la forma de expresarlo5 difiere en cuanto a su cualidad, ya que todo instinto puede llegar a ser impulso expresado y, cuando se expresa de manera reflexionada y armoniosa es estético, es poesía, es decir, es sutileza, delicadeza; acciones, palabras colores, que si cumplen con este atributo, tienen "elegancia... finura", en otras palabras: belleza. Por ello, en un estudio anterior (Patiño, 2014) comento que la acción, activada por la emoción, de acuerdo con Dewey, es acción intencional que busca la transformación de una situación que desequilibra, en una situación armoniosa. La emoción es dinamizadora y transformadora al mismo tiempo. El individuo, al ser impulsado a desplegar su creatividad, hace uso de sus recursos a fin de, mediante la acción, "corregir" o "ajustar" la situación detonante (p. 602).

Ahora bien, desde este punto de vista, es menester escuchar desde otra orilla la experiencia de la emoción, con sus significaciones y contingencias,

\footnotetext{
${ }^{5}$ Dewey distingue lo expresivo de la expresión. Del primero afirma que los seres humanos están en permanente expresividad, aunque no se den cuenta de ello, y lo ejemplifica diciendo que cuando un bebé llora es expresivo porque descarga directa e inmediatamente su inquietud en una acción ( $L W$ 10, p. 84). Ahora bien, la expresión se ha entendido como el tránsito de un sentimiento íntimo hacia afuera mediante una acción mecánica, o como una respuesta interna a una condición física (EW 4, p.157). Para Dewey, la expresión ha sido mediada por la comprensión y comunica una intención (LW 10, p. 67). La expresión ocurre cuando una emoción originaria e impulsiva se canaliza y transforma y consiste en desarrollar y resolver un problema. La definición más precisa de expresión es la que aparece en (EW 10, p. 67), es el resultado de haber convertido - mediante la reflexión y la significación-, un impulso -energía instintiva - en un acto creativo.
}

Hallazgos

ISSN: 1794-3841 | e-ISSN: 2422-409X | DOI: https://doi.org/10.15332/2422409X

Vol. 18 N.o 36| julio-diciembre del 2021 
desde la cotidianidad, desde lo poético o lo literario, que incluya el relato, la narración de cada individuo, quien en su afán de armonía da sentido y transforma mediante la acción (coordinada) su realidad. Esta perspectiva narrativa se encuentra en la apuesta rortiana que señala la necesidad de que se tenga en cuenta, con el fin de que efectúen, como lo señalo en Patiño (2018), "una influencia sobre nuestras acciones y sentimientos [...] que nos hacen conscientes sobre los efectos que nuestras acciones pueden llegar a tener en otras personas, ayudándonos a articular el sentimiento individual con [...] una utopía esperanzadora" (p. 93). Y es que para Rorty (1991), los relatos literarios y los demás profundizan y exploran lo afectivo

y lo emocional, lo que permite descubrir los pliegues de la experiencia vital individual del mundo, y romper así los límites de lo que nos ha sido heredado y enseñado a fin de pasar del mapa al territorio de la experiencia.

\section{Sobre la autora}

Martha Jeaneth Patiño Barragán. Doctora en Filosofía por la Pontificia Universidad Javeriana, graduada con Honores Magna Cum Laude, tesis doctoral laureada. Estudios de Maestría en Filosofía por la Universidad de Melbourne, Australia. Título de filósofa por la Universidad Nacional de Colombia, con estudios de Artes Plásticas en la Universidad de los Andes, de Escultura en la Arts Students League de Nueva York, de cerámica en el TAFE College, Melbourne, Australia. Actualmente es docente de planta de la Universidad Militar Nueva Granada, Colombia. Además de las tres publicaciones autocitadas en este artículo, es autora de los capítulos de libros: "La experiencia estética o la plena experiencia: El desplazamiento del espectador al artista en John Dewey” (2014); “El cuerpo: ese rasgo espacio-temporal y contingente de la experiencia. Un asunto de ritmos y tonos en el organicismo deweyano" (2015); una reseña del libro Estética analítica: entre el pragmatismo y el neopragmatismo 
(2015); así como de artículos académicos: "Hegel's Legacy” (2012);

"Dimensión religiosa de la vida cotidiana desde el proyecto filosófico de

John Dewey” (2019); “Nación, guerra y narración: la construcción del imaginario desde la negatividad necesaria de lo violento. Tres elementos para pensar a Colombia desde una obra de Fernando Soto Aparicio" (2020).

\section{Referencias}

Aristóteles. (1990). Retórica. Gredos.

Aristóteles. (1992). Acerca del alma. Gredos.

Aristóteles. (1993). Ética nicomáquea. Gredos.

Aristóteles. (1998). Política. Gredos.

Arnold, M. (1970). Perennial problems in the field of emotion. En M. Arnold (Ed). Feelings and emotions. The Loyola Symposyum (pp. 169-185). Academic Press New York.

Averill, J. (1980). A constructivist view of emotions. En R. Plutchik y H. Kellerman (Eds.) Emotion: theory, research and experience. Vol. 1 (pp. 305-339), Academic Press.

Bedford, E. (1956-1957). Emotions. Aristotelian Society, New Series, 57, 281-304.

Bericat, E. (2000). La sociología de la emoción y la emoción en sociología. Papers: Revista de Sociología, 62(62), 145-176. DOI: http://dx.doi.org/10.5565/rev/papers/v62no.1070

Calhoun, C. y Solomon, R. (Comps.) (1996). ¿Qué es una emoción? Lecturas clásicas sobre psicología filosófica. Fondo de Cultura Económica.

Dabrowski, A. (2016). Emotions in philosophy. A short introduction. Studia Humana, 5(3), 8-20. DOI: https://doi.org/10.1515/sh-2016-0011

Damasio, A. (1994). Descartes error. Putnam.

Damasio, A. (2009). En busca de Spinoza. Neurobiología de la emoción y los sentimientos. Crítica. 
Darwin, C. (2009). The expression of the emotions in man and animals. Cambridge University Press.

Descartes (1997). Las pasiones del alma. Tecnos.

Dewey, J. (1969-1972). The Early Works, 1882-1898. 5 vols. Southern Illinois University Press-Carbondale and Edwardsville.

Dewey, J. (1976-1983). The Middle Works, 1899-1924. 15 vols. Southern Illinois University Press-Carbondale and Edwardsville.

Dewey, J. (1981-1986). The Later Works, 1925-1953. 17 vols. Southern Illinois University Press-Carbondale and Edwardsville.

Elster, J. (2002). Alquimias de la mente. La racionalidad y las emociones. Paidós.

Frevert, U. (2011). History of emotions-Lost and found. Central European University Press.

Gadotti, M. (1998). Historia de las ideas pedagógicas. Siglo XXI.

Green, O. H. (1992). The emotions. Kluwer Academic Publishers.

Hillman, J. (1970). C. G. Jung's contributions to "feelings and emotions": Synopsis and implications. En M. Arnold (Ed). Feelings and emotions. The Loyola Symposyum (pp. 125-134) Academic Press.

Hochschild, A. (1983). The managed heart. Commercialization of human feeling. University of California Press.

James, W. y Lange, C. (1922). The emotions. Dunlap Knight.

James, W. (1950). Principles of psychology. Vols. I y II. Dover.

Kemper, T. (2011). Status, power and ritual interaction. A relational reading on Durkheim, Goffman and Collins. St. Johns University.

Kenny, A. (2003). Action, emotion and will. Routledge.

Knuutila, S. (2004). Emotions in ancient and medieval philosophy. Oxford University Press.

Lagier, D. (2009). Emociones, responsabilidad y derecho. Marcial Pons.

Le Breton, D. (1998). Las pasiones ordinarias. Antropología de las emociones. Nueva Visión.

Hallazgos

ISSN: 1794-3841 | e-ISSN: 2422-409X | DOI: https://doi.org/10.15332/2422409X

Vol. 18 N.o 36| julio-diciembre del 2021 
LeDoux, J. (1999). El cerebro emocional. Barcelona.

López, O. (2016, 13 de abril). De las emociones como categoría psicológica a las emociones como categoría socio-cultural [video]. YouTube. https://www.youtube.com/watch?v=S9AFjrs apo\&t=1027s

Lutz, C. (1998). Unnatural emotions: everyday sentiments on a Micronesian atoll and their challenge to western theory. University of Chicago Press.

Mesquita, B., De Leersnyder, J. y Boiger, M. (2016). The cultural psychology of emotions. En L. Feldman, M. Lewis y J. Havilland-Jones (Eds.). Handbook of emotions (pp. 393-411). The Guilford Press.

Mora, F. y Sanguinetti, A. (1994). Diccionario de neurociencias. Alianza.

Nussbaum, M. (2008). Paisajes del pensamiento. La inteligencia de las emociones. Paidós.

Papanicolaou, A. (2004). Walter Cannon y el surgimiento del cerebrocentrismo. Revista Española de Neuropsicología, 6(1-2), 25-52.

Patiño, M. (2011). Lo religioso. El sentido pleno de la experiencia en el proyecto filosófico de John Dewey. Pontificia Universidad Javeriana.

Patiño, M. (2014). Una aproximación pragmatista al problema del sufrimiento desde la práctica filosófica. En L. Cardona (Ed.), Filosofía y dolor: hacia la autocomprensión de lo humano (pp. 597-616). Pontificia Universidad Javeriana.

Patiño, M. (2018). La narrativa imaginativa como vía de acceso a la solidaridad. Una propuesta educativa desde el pragmatismo y el neo-pragmatismo. Hallazgos, 15(29), 87-109.

Rorty, R. (1991). Contingencia, ironía y solidaridad. Paidós.

Rosaldo, R. (1989). Cultura y verdad. Nueva propuesta de análisis social. Grijalbo.

Schachter, S. y Singer, J. (1962). Cognitive, social and physiological determinants of emotional state. Psychological Review, 69(5), 379-399.

Scheff, T. (1990). Micro-sociology, discourse, emotion and social structure. The University of Chicago Press.

Solomon, R. (2008). The philosophy of emotions. En L. Feldman, M. Lewis y J. Havilland-Jones (Eds.), Handbook of emotions (pp. 574-586). The Guilford Press.

Hallazgos

ISSN: 1794-3841 | e-ISSN: 2422-409X | DOI: https://doi.org/10.15332/2422409X

Vol. 18 N.o 36| julio-diciembre del 2021 
Taylor, G. (1985). Pride, shame and guilt. Emotions of self-assessment. Clarendon Press.

Vendrell, I. (2009). Teorías analíticas de las emociones: el debate actual y sus precedentes históricos. Contrastes. Revista Internacional de Filosofía, 14, 217-240.

Hallazgos

ISSN: 1794-3841 | e-ISSN: 2422-409X | DOI: https://doi.org/10.15332/2422409X

Vol. 18 N. 0 36| julio-diciembre del 2021 